\title{
一些模型问题高阶离散格式的 低阶预条件
}

\author{
黄 维 章 \\ (中国科学院应用数学研究所, 北京 100080)
}

关钦高阶格式、低阶格式、预条件

\section{一、引 声}

偏微分方程的高阶 (湤做)格式能以较少的节点而获得高精度的离散解, 但所形成的代数 系统却非常病态和具有许, 的非零元. 为了有效地求解此代数系统, Wong $g^{[1.2]}$ 提㟧了使用低

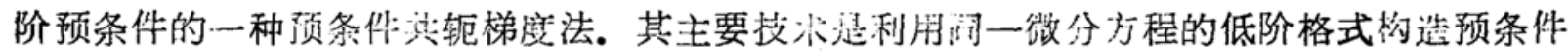
矩阵. 此技术的有效性已由一些数值实验所证实, 却且在谱多望网格法 ${ }^{[3]}$ 中得到了重要应用.

目前,这蚛低阶顸条件技术仍停留在数值研究上. 要作琶沦研究,首先要回答如下两个间 题：（1）根据低阶格式构造的预条件矩阵，对低阶格式与对高阶格式的有效性之间有何联系? (2)根据同一微分方程的所有低阶格式构造的有效预条件哑汗, 是不是对高阶格式都育效?

本文针对二维和三维的槛型问题，通过 Fourier 分析,给出了一些低阶和高阶格式的有效 性之间的内在联系,并对问题(2)给出了否定的回答, 即根据某些低阶格式构造的有效预条件 矩阵对高阶格式是很有效的, 而根据号外一些呧阶格式芶造的有效(对低阶格式而言)预条件 矩阵却对高阶格式元效。

\section{二、一些术语和记号}

假定 $L$ 是微分算子, $\Omega \subset \mathbb{R}^{d}, L_{h}$ 是给定在 $Q$ 的某网格 $\Omega_{k}$ 上的差分算子, 其中 $h$ 是表征 网格粗细的参数.

定义 2.1 说差分算子 $L_{b}$ 在节点 $x^{i} \in \Omega_{k}$ 以阶 $p$ 逼近 $L$, 如果当 $h \rightarrow 0$ 时 $\phi_{i}-L_{k} v_{i}-$ $(L v)_{i}=O\left(h^{p}\right)$, 其中 $v(x)$ 是充分光滑的函数. 如果 $L_{k}$ 在所有节点 $x^{i} \in \Omega_{b}$ 上都以阶 $P$ 逼近 $L$, 则说 $L_{k}$ 在网格 $Q_{k}$ 上 $p$ 阶逼近 $L$, 或简称 $L_{k}$ 有 $p$ 阶精度.

定义 2.2 假定有一个微分方程 $L u=+f(x)$, 在 $\Omega_{k}$ 上用差分方程 $L_{k} y=+\varphi(x)$ 逼 近. 如果对某种范数 $\|\cdot\|,\left\|L_{h} u-\varphi(x)\right\|=O\left(h^{p}\right)$, 其中 $u$ 为微分方程的解, 则说差分方程以 阶 $p$ 按范数 $\|\cdot\|$ 逼近微分方程, 或简称差分方程有 $p$ 阶精菨.

本文 1990 年 11 月5 日收到. 1991 年 11 月 24 日收到修没稿.

- 中国科学院数学特别支持费资助课题.

第: 期 
在以下讨论差分方程的精度时, 均假定使用范数 $\|\cdot\|=\max _{o_{n}} \mid \cdot 1$. 下面引人几个微分、差 分算子。

$$
\begin{gathered}
L_{a} v=-\frac{\partial^{2} v}{\partial x_{a}^{2}}, \alpha=1, \cdots, d_{0} \\
\Lambda_{\alpha} \nu=\frac{-v\left(\cdots, x_{a}+h, \cdots\right)+2 v\left(\cdots, x_{a}, \cdots\right)-v\left(\cdots, x_{a}-h, \cdots\right)}{h^{2}} \\
\alpha=1, \cdots, d .
\end{gathered}
$$

那么对于足够光滑的函数 $\nu$, 有

$$
\begin{gathered}
\Lambda_{a} v=-\frac{\partial^{2} v}{\partial x_{a}^{2}}-\frac{h^{2}}{12} \frac{\partial^{4} v}{\partial x_{a}^{4}}-\frac{h^{4}}{360} \frac{\partial^{6} v}{\partial x_{a}^{6}}+O\left(h^{6}\right) . \\
\text { 三、二维模型问题 }
\end{gathered}
$$

考虑二维 Poisson 方程的 Dirichlet 问题

$$
\left\{\begin{array}{l}
-\Delta u=f, \text { 在 } Q=(0,1) \times(0,1) \text { 内; } \\
u=0, \text { 在 } \partial \Omega \text { 上, }
\end{array}\right.
$$

其中总假定 $f$ 足够光滑. 对某正整数 $n$, 令 $h=\frac{1}{n+1}$,

$$
\Omega_{k}=\left\{\left(x_{1, i}, x_{2, j}\right)=(i h, j h): i, j=1, \cdots, n\right\} .
$$

表 1 给出了问题 (3.1) 的常用几种差分格式及其特点, 其中特征网格函数 $\phi_{p . q}(p, q=$ $1, \cdots, n)$ 为

以及特征值

$$
\left(\phi_{p, q}\right)_{i, j}=\sin p \pi x_{1, i} \sin q \pi x_{2, j}, i, j=0, \cdots, n+1
$$

\begin{tabular}{|c|c|c|c|c|c|c|c|}
\hline 墓 & 分 & 格 & \multicolumn{2}{|c|}{ 相应代数系统 } & \multirow{2}{*}{$\begin{array}{l}\text { 饡分 } \\
\text { 精度 }\end{array}$} & \multirow{2}{*}{$\begin{array}{l}\text { 差分 } \\
\text { 方程 } \\
\text { 精 }\end{array}$} & \multirow{2}{*}{$\begin{array}{l}\text { 系数矩阵相应于 } \\
\phi_{,, 4} \text { 的特征值 }\end{array}$} \\
\hline 差分算子 & & $\varphi$ & $\begin{array}{l}\text { 系数 } \\
\text { 矩阵 }\end{array}$ & $\begin{array}{l}\text { 非繁对 } \\
\text { 角线数 }\end{array}$ & & & \\
\hline$\Lambda_{1}+\Lambda_{2}$ & & $f$ & $A$, & 5 & 2 阶 & 2 阶 & $\lambda_{p}+\lambda_{a}$ \\
\hline$\Lambda_{1}+\Lambda_{2}-\frac{h^{2}}{2} \Lambda_{1} \Lambda_{2}$ & & $f$ & $A_{5}^{*}$ & 5 & 2 阶 & 2 阶 & $\lambda_{b}+\lambda_{q}-\frac{h^{2}}{2} \lambda_{D} \lambda_{Q}$ \\
\hline$\Lambda_{1}+\Lambda_{2}-\frac{k^{2}}{6} \Lambda_{1} \Lambda_{2}$ & & $f+\frac{h^{2}}{12} \Delta f$ & $A_{9}$ & 9 & 2 阶 & 4 阶 & $\lambda_{D}+\lambda_{Q}-\frac{h^{2}}{6} \lambda_{p} \lambda_{Q}$ \\
\hline$\Lambda_{1}+\Lambda_{2}-\frac{h^{2}}{6} \Lambda_{1} \Lambda_{2}$ & $t+$ & $\frac{h^{2}}{12} \Delta f+\frac{h^{4}}{360}\left(\Delta^{2} f+2 L_{1} L_{2} f\right)$ & $A_{9}$ & 9 & 2 阶 & 6 阶 & $\lambda_{p}+\lambda_{q}-\frac{h^{2}}{6} \lambda_{p} \lambda_{q}$ \\
\hline
\end{tabular}

$$
\lambda_{p}=\frac{4}{h^{2}} \sin ^{2} \frac{p g h}{2}
$$

容易发现,前面三种格式具有关系

$$
A_{3}=\frac{2}{3} A_{5}+\frac{1}{3} A_{3}^{*} .
$$

根据条件数的定义和表 $1,(3.4)$ 容易得到

表 1 问题(3.1)的几种差分格式及其特点 
引理 3.1 对于任意两个对称正定矩阵 $A, B \in \mathbb{R}^{n \times n}$, 有

$$
\max \left\{\frac{K(A)}{K(B)}, \frac{K(B)}{K(A)}\right\} \leqslant K(A B) \leqslant K(A) K(B) .
$$

引理 3.2 $0 \leqslant \frac{\lambda_{p}+\lambda_{q}-\frac{h^{2}}{2} \lambda_{p} \lambda_{q}}{\lambda_{p}+\lambda_{q}} \leqslant 1, p, q-1, \cdots, n$.

引理 3.3 当 $h \rightarrow 0$ 时, 有

$$
\max _{p . q} \frac{\lambda_{p}+\lambda_{q}}{\lambda_{p}+\lambda_{q}-h^{2} / 2 \lambda_{p} \lambda_{q}}-\frac{4}{\pi^{2}} h^{-2}+O\left(h^{-1}\right) \text { 和 } \min _{p . q} \frac{\lambda_{p}+\lambda_{q}}{\lambda_{p}+\lambda_{q}-h^{2} / 2 \lambda_{p} \lambda_{q}}=1+O(h) \text {. }
$$

在上面引理中, $K(A)$ 表示 $A$ 的条件数. 假定 $P$ 是任意的对称正定矩阵, 那么根据 $(3.5)$ 及 相似矩阵特征相等, 我们有

$$
\begin{aligned}
K\left(P^{-1} A_{9}\right) & =K\left(P^{-1} A_{5}^{1 / 2}\left[\frac{2}{3} I+\frac{1}{3} A_{5}^{-1 / 2} A_{5}^{*} A_{5}^{-1 / 2}\right] A_{5}^{1 / 2}\right) \\
& =K\left(A_{5}^{1 / 2} P^{-1} A_{5}^{1 / 2}\left[\frac{2}{3} I+\frac{1}{3} A_{5}^{-1 / 2} A_{5}^{*} A_{5}^{-1 / 2}\right]\right) .
\end{aligned}
$$

同理,有

$$
K\left(P^{-1} A_{9}\right)=K\left(A_{5}^{* 1 / 2} P^{-1} A_{5}^{* 1 / 2}\left[\frac{2}{3} A_{5}^{*-1 / 2} A_{5} A_{5}^{*-1 / 2}+\frac{1}{3} I\right]\right) .
$$

注意到 $A_{5}^{-1 / 2} 、 A_{5}^{*} 、 A_{5}$ 和 $A_{5}^{*-1 / 2}$ 有相同的特征向量系, 故引理 3.1-3.3 和(3.7)、(3.8)可推出

定理 3.1 对任意的对称正定矩阵 $P$, 有

$$
\frac{2}{3} K\left(P^{-1} A_{5}\right) \leqslant K\left(P^{-1} A_{9}\right) \leqslant \frac{3}{2} K\left(P^{-1} A_{5}\right) 。
$$

定理 3.2 对任意的对称正定矩阵 $P$, 当 $h \rightarrow 0$ 时有

$$
K\left(P^{-1} A_{9}\right) \geqslant\left[\frac{8}{3 \pi^{2}} h^{-2}+O\left(h^{-1}\right)\right] / K\left(P^{-1} A_{\xi}^{*}\right) .
$$

定理 3.1 和 3.2 给出了任意预条件矩阵 $P$ 对不同格式的预条件效率之间的关系. 定理 3.1 说明, 若 $P$ 根据低阶格式 (二阶五点格式)构造且很有效 (例如 $K\left(P^{-1} A_{\xi}\right) \sim 1$ ), 那么 $P$ 对高 阶格式 (四阶或六阶九点格式)也非常有效 $\left(K\left(P^{-1} A_{9}\right) \$ 3 / 2\right)$. 反之, 定理 3.2 却说明, 即使 $K\left(P^{-1} A_{*}^{*}\right)$ 很小 (例如, $\left.K\left(P^{-1} A_{s}^{*}\right) \sim 1\right)$, 但 $P$ 也不可能成为高阶格式(这里为九点格式)的有 效预条件矩阵.

\section{四、三维模型问题}

考虑三维 Poisson 方程的 Dirichlet 问题

$$
\left\{\begin{array}{l}
-\Delta u=f, \text { 在 } Q=(0,1) \times(0,1) \times(0,1) \text { 内; } \\
u=0, \quad \text { 在 } \partial Q \text { 上, }
\end{array}\right.
$$

其中假定 $f$ 足够光滑. 令 $h=\frac{1}{n+1}, n$ 为正整数, $\Omega_{b}$ 为立方体网格. 表 2 给出了(4.1)式 的三种差分格式及其特点, 其中 $\lambda_{i}(i=p, q, r)$ 见(3.4)式,特征函数 $\phi_{p, q, r}$ 为

$$
\left(\phi_{p, q, r}\right)_{i, j, k}=\sin p \pi x_{1, i} \sin q \pi x_{2, j} \sin r \pi x_{3, k}, i, j, k=0,1, \cdots, n+1 \text {. }
$$

类似 $\$ 3$, 根据引理 3.1 和表 2 , 我们有

第 7 期

科学通报 
表 2 问题 (4.1)的三种差分格式及其特点

\begin{tabular}{|c|c|c|c|c|c|c|}
\hline \multicolumn{2}{|c|}{ 差 分 格 式 } & \multicolumn{2}{|c|}{ 相应代数系统 } & \multirow{2}{*}{$\begin{array}{l}\text { 差分 } \\
\text { 尊子 } \\
\text { 精变 }\end{array}$} & \multirow{2}{*}{$\begin{array}{l}\text { 差分 } \\
\text { 方程 } \\
\text { 精 }\end{array}$} & \multirow{2}{*}{$\begin{array}{l}\text { 系数矩阵相应于 } \\
\phi_{\text {D.4., 的特征值 }}\end{array}$} \\
\hline 差分配子 & $\varphi$ & $\begin{array}{l}\text { 系数 } \\
\text { 矩阵 }\end{array}$ & $\begin{array}{l}\text { 非零对 } \\
\text { 角线数 }\end{array}$ & & & \\
\hline$\Lambda_{1}+\Lambda_{2}+\Lambda_{3}$ & $f$ & $A$, & 7 & 2 阶 & 2 阶 & $\lambda_{p}+\lambda_{a}+\lambda_{r}$ \\
\hline $\begin{array}{l}\Lambda_{1}+\Lambda_{2}+\Lambda_{3}- \\
\frac{h^{2}}{6}\left(\Lambda_{1} \Lambda_{2}+\Lambda_{1} \Lambda_{3}+\Lambda_{2} \Lambda_{3}\right)\end{array}$ & $f+\frac{h^{2}}{12} \Delta f$ & $A_{1}$ & 19 & 2 阶 & 4 阶 & $\begin{array}{l}\lambda_{D}+\lambda_{Q}+\lambda_{r}- \\
\frac{h^{2}}{6}\left(\lambda_{p} \lambda_{Q}+\lambda_{p} \lambda_{r}+\lambda_{q} \lambda_{r}\right)\end{array}$ \\
\hline $\begin{array}{l}\Lambda_{1}+\Lambda_{2}+\Lambda_{3}- \\
\frac{h^{2}}{6}\left(\Lambda_{1} \Lambda_{2}+\Lambda_{1} \Lambda_{3}+\Lambda_{2} \Lambda_{3}\right) \\
\quad+\frac{h^{4}}{30} \Lambda_{1} \cdot \Lambda_{1} \Lambda_{3}\end{array}$ & $\begin{array}{l}f+\frac{h^{2}}{12} \Delta f+\frac{h^{4}}{360}\left(\Delta^{2}+\right. \\
2 L_{1} L_{2}+2 L_{1} L_{3} \\
\left.+2 L_{2} L_{3}\right) f\end{array}$ & $A_{21}$ & 27 & 2 阶 & 6 阶 & $\begin{array}{l}\lambda_{D}+\lambda_{a}+\lambda_{r}- \\
\frac{h^{2}}{6}\left(\lambda_{0} \lambda_{q}+\lambda_{0} \lambda_{r}+\lambda_{q} \lambda_{r}\right) \\
\quad+\frac{h^{4}}{30} \lambda_{v} \lambda_{q} \lambda_{r}\end{array}$ \\
\hline
\end{tabular}

定理 4.1 对任意的对称正定矩阵 $P$,有

$$
\begin{aligned}
& \frac{1}{3} K\left(P^{-1} A_{7}\right) \leqslant K\left(P^{-1} A_{1}\right) \leqslant 3 K\left(P^{-1} A_{1}\right), \\
& \frac{23}{45} K\left(P^{-1} A_{7}\right) \leqslant K\left(P^{-1} A_{27}\right) \leqslant \frac{45}{23} K\left(P^{-1} A_{7}\right) .
\end{aligned}
$$

定理 4.1 给出了七点格式与十九点格式和七点格式与二十七点格式之间的预条件效率的 关系.

最后需说明的是, 在 (4.3) 和 (4.4) 式中, 实际上当 $h \rightarrow 0$ 时有 $K\left(P^{-1} A_{1^{y}}\right) \approx 3 K\left(P^{-1} A_{7}\right)$ 和 $K\left(P^{-1} A_{27}\right) \approx \frac{45}{23} K\left(P^{-1} A_{7}\right)$. 在 Wong ${ }^{[1]}$ 中, 已计算出 $K\left(P^{-1} A_{7}\right)^{1)}-7.71(n-19), 12.20$ $(n-29)$ 以及 $K\left(P^{-1} A_{27}\right)=15.64(n-19), 23.98(n-29)$, 其中 $P$ 为七点格式的行和一 致预条件矩阵. 而按此文的诏计, 则有 $K\left(P^{-1} A_{11}\right)=15.09(n-19), 23.88(n-29)$. 这 两组数据是非常吻合的.

\section{参考文献}

[1] Wong, Y. S., Intern. J. Computer Math., 19 (1986), 177-200.

[2] - ibid., 23 (1988), $141-165$.

[3] Heinrichs, W., Notes on Numerical Fluid Mechanics, Vieweg, 23 (1939), 139-144.

1) Wong 的这些计算侸均有一定的计算误差. 